\title{
Journal of Oncology and Cancer Research (JOCR)
}

\section{Objectively Recorded Physical Activity And Perceived Distress At The Beginning Of Oncological Treatment}

Anja Rangen ${ }^{1}$, Karin Nordin ${ }^{1,2}$, Cecilia Arving ${ }^{2,3}$, Inger Thormodsen ${ }^{3},{ }^{*}$ Sveinung Berntsen ${ }^{1,2}$

\footnotetext{
${ }^{1}$ Department of Public Health, Sport and Nutrition; Faculty of Health and Sport Sciences; University of Agder, Kristiansand, Norway

${ }^{2}$ Department of Public Health and Caring Sciences, Uppsala University, Uppsala, Sweden

${ }^{3}$ Department of Oncology and Medical Physics, Haukeland University Hospital, Bergen, Norway
}

Received 15/02/2017

Accepted 23/05/2017

Published 19/06/2017

\section{*For Correspondence}

Sveinung Berntsen

Department of Public Health, Sports and Nutrition, Faculty of Health and Sport Sciences

University of Agder

Postal address: Post Box 422, 4604 Kristiansand, Norway

Contact no: +4738141045

Email: sveinung.berntsen@uia.no

Keywords: Activity Monitor, Cancer, Distress, During Treatment, Physical Activity

\section{Research Article}

\begin{abstract}
Purpose: To examine the association between physical activity (PA), objectively recorded as number of steps and hours of moderate- tovigorous intensity PA, and self- reported distress (anxiety, depression, intrusion and avoidance) at the beginning of oncological treatment. Methods: In the present prospective study 244 cancer patients provided data on PA and distress at the beginning of oncological treatment, including measures from baseline and six weeks later. Results: At baseline, 16 patients with depression walked significantly fewer steps per day, compared to patients without depression $(P=0.035)$. The same tendency was seen for moderate- to- vigorous intensity $P A$, although not significant $(P=0.077)$. At six weeks, there was no association between depression and baseline PA. Anxiety, intrusion and avoidance were not associated with PA. Conclusion: An inverse association between PA and depression at baseline was found, especially in relation to number of walking steps. This finding must be interpreted with caution due to few patients with depression. Whether a physically active lifestyle prevents or reduces distress when diagnosed with cancer should be elaborated in future studies.
\end{abstract}




\section{INTRODUCTION}

Cancer is an increasingly prevalent disease and a growing problem which requires long term management ${ }^{[1]}$. A cancer diagnosis and treatment might entail a demanding adjustment process and disruption in important areas of life, possibly causing distress ${ }^{[2]}$. Distress has been reported in up to two- third of cancer patients, and might be present from diagnosis to several years after treatment is completed ${ }^{[2,3,4]}$. Highest prevalence of distress is seen at diagnosis with a significant decrease throughout the following year ${ }^{[3]}$. Distress may affect cancer patients' lives in several ways, e.g., impaired quality of life and reduced probability of making positive changes in health behavior, such as physical activity ${ }^{[5]}$.

Physical activity seems to be both feasible and safe during cancer treatment ${ }^{[6]}$. Still many cancer patients reduce their physical activity level significantly during treatment, and do not regain to their former level of physical activity after treatment is completed ${ }^{[7]}$. Whether a patient is physically active at diagnosis has been shown as a strong predictor of whether that individual will be physically active during treatment ${ }^{[8]}$.

In the general population, physical activity has a positive impact on distress ${ }^{[9]}$. There are indications that physical activity may influence distress positively in cancer patients both during and after oncological treatment ${ }^{[10,11]}$. For adult cancer patients at the start of treatment, higher self- reported physical activity levels have been associated with reduced anxiety and depression ${ }^{[12,13]}$. In cohorts and in a retrospective study, it has been suggested that being physically active before cancer diagnosis and during early treatment may result in less depression ${ }^{[14,15,16]}$. However, previous studies have mostly used self-reported measures of physical activity or included patients with breast cancer. To our knowledge, no studies have yet examined the association between objectively registered physical activity and distress at the beginning of oncological treatment in a heterogeneous group of cancer patients.

The aim of the present study was to examine the association between objectively recorded physical activity (average number of steps per day and hours of moderate- to- vigorous intensity physical activity) and selfreported distress (anxiety, depression, intrusion and avoidance) at the beginning of oncological treatment.

\section{METHODS}

\section{Design}

The present study is part of a larger study investigating the effect of an individually administered stepped-care stress management intervention, including encouragement to be physically active ${ }^{[17]}$. The present study used a prospective design with data from beginning of oncology treatment including measures at baseline and 6 weeks later. At baseline, the number of walking steps and hours of moderate-to-vigorous intensity physical activity per day were registered using an activity monitor. Distress in terms of anxiety, depression, intrusion and avoidance were registered at both time points using questionnaires. The project was approved by the Regional Committee for Medical and Health Research Ethics and the Data Inspectorate of Norway (2010/1911).

\section{Patients and recruitment}

Patients were included between May 2011 and June 2013. Inclusion criteria were 1) age above 18 years; 2) newly diagnosed with breast-, prostate-, testicle-, lymphoma or colorectal cancer; 3 ) scheduled for cancer treatment by neo-adjuvant, adjuvant or other potentially curative treatment (chemotherapy, radiation therapy or hormonal 
therapy, given separately or in a combination); 4) treated at the Department of Oncology and Medical Physics at Haukeland University Hospital, Bergen, Norway. Exclusion criteria were a prior cancer diagnosis, ongoing psychiatric condition or insufficient Norwegian language skills.

A total of 677 patients fulfilled the inclusion criteria and were consecutively asked to participate. When patients received information about the cancer treatment at the clinic, eligible patients were first contacted by mail informing them about the study and that a project staff member would contact them by telephone within a week asking them to participate in the study. During the phone call patients received information about the study aim and methods, and were also given the opportunity to ask questions. After this 384 patients decided not to participate in the study and reasons for this were no need for psychosocial intervention, too far to travel, reluctance to participate in research, too stressful, and being too old or too tired. The 293 patients (43\%) who accepted participation received baseline questionnaires, written instructions and a request for written informed consent by mail together with a prepaid return envelope. Staff members contacted participants who had given incomplete responses in their questionnaires to ask them to complete the questionnaire. To ensure a high response rate a reminder was sent to the patients who had not returned their questionnaires within 14-21 days. The participants were excluded from the present study if they did not return the questionnaires after three reminders. A total of 291 patients returned the baseline questionnaires and received a physical activity monitor by mail with instructions to use it for seven consecutive days. The present study consisted of the 244 patients (84\%) who wore the activity monitor sufficiently to get at least one valid day with physical activity data, these 244 participants are included in the analysis.

\section{MEASURES}

\section{Demographic data}

The following variables were collected through a questionnaire: Age, gender, body mass $(\mathrm{kg})$, height $(\mathrm{cm})$, marital status, person per household, education level, annual income, main income source, smoking- and alcohol consumption, cancer diagnosis and perceived social support. Body mass index (BMI) was calculated $\left(\mathrm{kg} / \mathrm{m}^{2}\right)$.

\section{Distress}

In the present study, psychological distress in terms of anxiety and depression (HADS-scale) and subjective distress in terms of intrusion and avoidance (IES-scale) was measured.

The Hospital Anxiety and Depression Scale (HADS) is a 14 item self- reported questionnaire divided in two subscales, anxiety and depression. Each subscale consists of 7 items and measures the presence and severity of symptoms during the past week. Every item has ranged from 0 (no problem) to 3 (severe problem), maximum score for each of the two subscales are thus 21. Recommended cut- off scores for each of the two subscales was 7 or less for no cases (no anxiety/-depression) and 8 or more for doubtful/definite cases (anxiety/-depression). The HADS is suited among patients with somatic illness ${ }^{[18]}$. HADS is valid in cancer patients ${ }^{[19]}$.

The Impact of Event Scale (IES) is a 15-item self-reported questionnaire to measure frequencies of subjective distress during the past week. It is divided in two subscales, intrusion ( 7 items) and avoidance (8 items). These include intrusive images and thoughts and denial/-avoidance behavior. The frequency of intrusion and avoidance were estimated for each item on a scale ranging from 0 (not at all) to 5 (often). Maximum score for intrusion items are 35 and for avoidance $40^{[20]}$. Recommended cut-off for each of the subscales was low (=8) (no intrusion/avoidance) and medium/high ( $\geq 9$ ) (intrusion/-avoidance) ${ }^{[21]}$. The psychometric properties of the IES are satisfactory ${ }^{[22]}$. 


\section{Physical activity}

Physical activity level was recorded objectively using the SenseWear ${ }^{\mathrm{TM}}$ Pro3 Armband (SWA) (BodyMedia Inc., Pittsburgh, PA, USA) according to the manufacturer. The SWA is valid for use during free-living activities ${ }^{[23]}$, also among cancer patients ${ }^{[24]}$. Sense Wear Professional Research Software (V. 6.1, Body Media) developed by the manufacturer was used to download and analyze data from the monitor. Only days from recordings consisting of at least 19.2 hours (80\%) per day were defined as a valid day and included in the analysis. Data from SWA describing physical activity are average number of steps per day and average hours of moderate-to-vigorous intensity physical activity per day. Cut-off defining moderate-to-vigorous intensity physical activity was 3 metabolic equivalents (METS) according to Garber et al. ${ }^{[25]}$.

\section{Statistical analysis}

Data was analyzed using the Statistical Package for the Social Sciences (SPSS), version 22 (International Business Machines Corporation (IBM), New York, United States). Statistical significance level was set to $5 \%$. Differences between groups were analyzed by Chi-square and Man-Whitney $U$ test, and differences within groups were analyzed by Wilcoxon Signed Rank test.

Logistic regression analyses were applied with the dependent variables (low/high) intrusion and avoidance (IES), and (non-case/case) anxiety (HADS) measured at baseline and after 6 weeks. Due to multicollinearity distress at baseline were not included in the models with distress after 6 weeks at dependent variable. Analysis was conducted in 6 separate models based on recommended cut-off points for respectively dependent variable. Due to multicollinearity, moderate-to-vigorous intensity physical activity and walking steps were not included in the same models. Diagnosis was excluded in favor of gender, and marital status was excluded in favor of person per household due to multicollinearity. The final multivariate model was built as described by Hosmer and Lemeshow

${ }^{[26]}$. Walking steps and moderate-to-vigorous intensity physical activity were not removed from the model. Due to a limited number of patients with depression, descriptive bivariate analyses were carried out for this outcome variable.

\section{RESULTS}

There were no significant differences in demographics between participants with and without valid physical activity data, except from height $(P=0.015)$ (table 1 ). 
Table 1 Background data presenting the participants by valid physical activity data. Data are given as number (\%) unless otherwise stated.

\begin{tabular}{|c|c|c|c|}
\hline & $\begin{array}{l}\text { Not valid SW data } \\
\qquad(N=47)\end{array}$ & $\begin{array}{l}\text { Valid SW data } \\
\qquad(\mathrm{N}=244)\end{array}$ & P-values ${ }^{a}$ \\
\hline Age (years); mean (SD) & $60.8(11.2)$ & $61.0(10.6)$ & 0.914 \\
\hline \multicolumn{4}{|l|}{ Gender } \\
\hline Female & $25(53)$ & $112(46)$ & 0.359 \\
\hline Height (cm); mean (SD) & $170(8)$ & $173(8)$ & 0.015 \\
\hline Weight (kg); mean (SD) & 77 (11) & $78(14)$ & 0.373 \\
\hline BMI; mean (SD) & $26(3)$ & $26(4)$ & 0.419 \\
\hline \multicolumn{4}{|l|}{ Marital status } \\
\hline Married/ cohabited & 37 (79) & $191(78)$ & 0.946 \\
\hline \multicolumn{4}{|l|}{ Number of persons per household* } \\
\hline$\geq 2$ persons & $38(81)$ & $203(83)$ & 0.566 \\
\hline $\begin{array}{l}\text { Households total income per year } \\
\text { (Per } 100.000 \text { n.kr); Median (IQR) }\end{array}$ & $6.5(5.5)$ & $6.0(4.1)$ & 0.584 \\
\hline \multicolumn{4}{|l|}{ Educational level } \\
\hline Elementary/secondary school & $30(64)$ & $130(53)$ & 0.183 \\
\hline \multicolumn{4}{|l|}{ Main income source* } \\
\hline Paid Labour & $15(32)$ & $84(34)$ & 0.734 \\
\hline Social Security etc. & $15(32)$ & $64(26)$ & \\
\hline Other & $17(36)$ & $95(39)$ & \\
\hline \multicolumn{4}{|l|}{ Smoking } \\
\hline Daily & $6(13)$ & $22(9)$ & 0.425 \\
\hline \multicolumn{4}{|l|}{ Alcohol consumption* } \\
\hline$\geq 2$ times/week & $12(26)$ & $67(28)$ & 0.774 \\
\hline \multicolumn{4}{|l|}{ Cancer diagnoses } \\
\hline Breast cancer & $22(47)$ & $101(41)$ & 0.763 \\
\hline Prostate cancer & $19(40)$ & $105(43)$ & \\
\hline Other & $6(13)$ & $38(16)$ & \\
\hline \multicolumn{4}{|l|}{ Perceived social support* } \\
\hline Have social support & $25(53)$ & $117(48)$ & 0.503 \\
\hline
\end{tabular}

a $\mathrm{P}$ - values indicates differences between groups. Statistic significant values are given in bold. *Variables with missing number of data; $\mathrm{n}=1-5$. SD; Standard deviation. IQR; interquartile range. Cm; centimeter. Kg; kilograms. BMI: Body mass index. N.kr; Norwegian kroners. 
Median values for levels of depression increased significantly $(P=0.041)$ from baseline to six weeks. For anxiety, intrusion and avoidance there were no significant changes in median distress levels from baseline to six weeks (table 2). Number of patients with depression $(n=16)$ did not change from baseline to six weeks; however, seven patients were depressed at both measures (not necessarily the same individuals), and 18 were depressed at one point only. For anxiety, intrusion and avoidance, prevalence of distress at baseline was $14 \%, 32 \%$ and $45 \%$, respectively, each increasing by $2 \%$ from baseline to six weeks (results not shown).

Table 2 Self- reported intrusion, avoidance, anxiety and depression at baseline and six weeks later, for all patients, median (IQR)
Baseline
6 weeks
P- value

\begin{tabular}{r|c|c|c|}
\hline Intrusion & $6(8)$ & $6(9)$ & 0.899 \\
\hline Avoidance & $8(10)$ & $8(11)$ & 0.135 \\
\hline Anxiety & $3(5)$ & $3(6)$ & 0.997 \\
\hline Depression & $1(3)$ & $2(3)$ & $\mathbf{0 . 0 4 1}$ \\
\hline
\end{tabular}

\# P- values indicates a significant increase in depression from baseline to 6 weeks (bold). Missing number of data; $n=1-8$.

The 16 patients with depression were walking significantly less steps $(P=0.035)$ per day compared to patients without depression, at baseline (median (IQR) 4467 (6334) steps and 7256 (4682) steps for the two groups, respectively. The same tendency was seen for moderate- to- vigorous intensity physical activity, although not significant ( $P=0.077$ ) (fig.1. $a$ and $c)$.

At six weeks, there was no longer a significant association or tendency between baseline physical activity and depression ( $\mathrm{P}=0.253$ for walking steps and $\mathrm{P}=0.840$ for moderate-to-vigorous intensity physical activity, respectively) (fig. 1. $b$ and d). In multivariate logistic regression analysis, there were no significant associations between moderate- to- vigorous intensity physical activity or number of walking steps, and intrusion, avoidance and anxiety, at neither baseline nor six weeks later (Appendix 1-6). 
a.

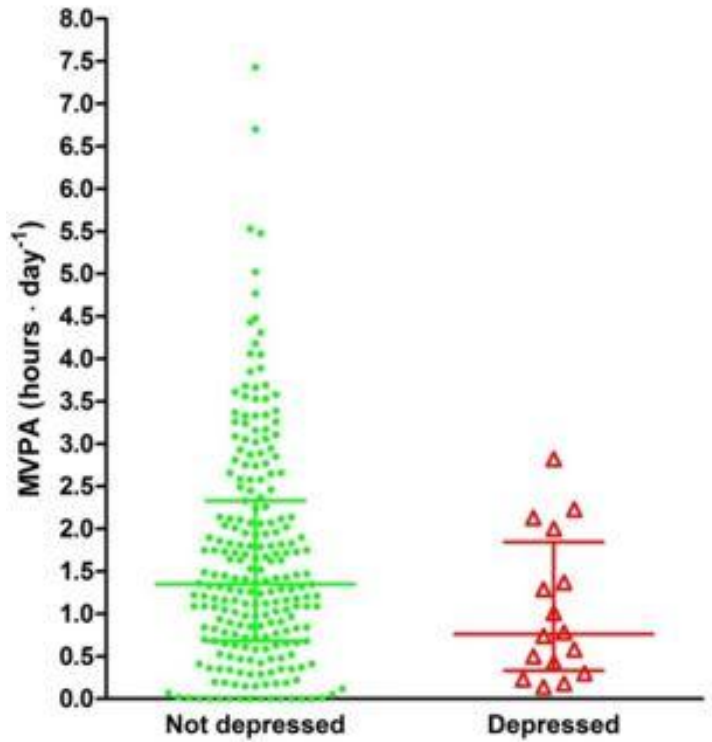

c.

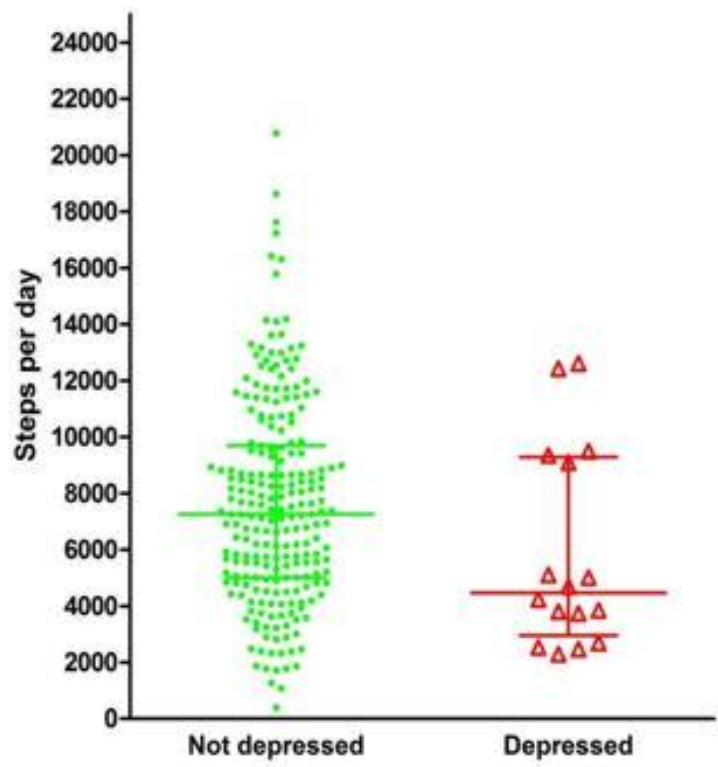

b.

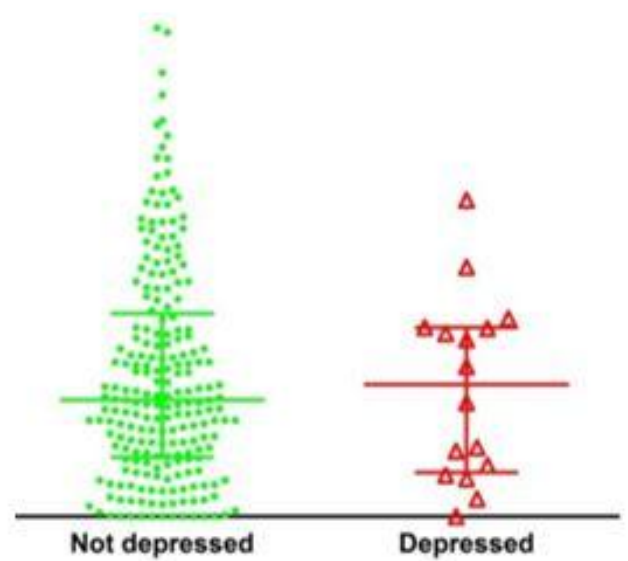

d.

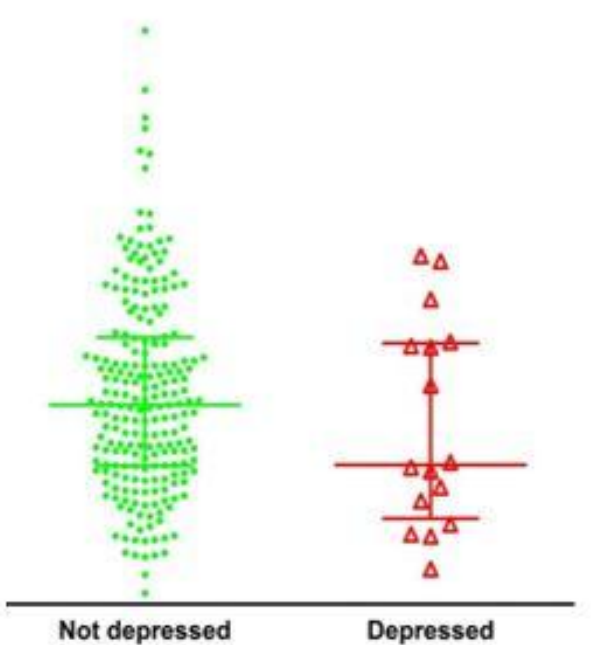

Fig. 1. Hours of moderate- to- vigorous intensity physical activity (MVPA) and steps per day for patients with and without depression (median (IQR)): MVPA (a) at baseline (b) at six weeks.

Number of steps (c) at baseline (d) at six weeks 


\section{DISCUSSION}

In the present prospective study of $\mathbf{2 4 4}$ cancer patients, the number of walking steps was significantly associated with lower levels of depression at the very beginning of oncological treatment. The same tendency was seen for moderate- to- vigorous intensity physical activity, although not significant. For anxiety, intrusion and avoidance we did not find any association with physical activity.

To our knowledge, this is the first study to explore the association between objectively recorded MVPA and walking steps and distress among cancer patients at the beginning of oncological treatment. The findings that the number of steps was associated with lower levels of depression are supported by studies in the general population, where number of steps were assessed using a step counter ${ }^{[27]}$. Previous findings regarding moderate-to-vigorous intensity physical activity and psychological distress among cancer patients at the beginning of treatment are contrary to the results in the present study. In a randomized-controlled trial a combination of self-directed stress management training and home-based exercise increased self-reported physical activity levels and reduced depression and anxiety among 286 cancer patients, mainly diagnosed with breast and lung cancer, at the start of chemotherapy ${ }^{[13]}$. A similar tendency was seen in a cross- sectional study among 240 women with breast cancer, where an association between higher self-reported physical activity levels and reduced depression was found 2-10 weeks post-surgery ${ }^{[12]}$. In a cross-sectional study among 180 colon cancer survivors objectively recorded moderate-to-vigorous intensity physical activity was associated with anxiety but not depression ${ }^{[28]}$. However, these patients had finished their treatment and are therefore not directly comparable to our patients in the beginning of their oncological treatment. To our knowledge, the association between PA and intrusion or avoidance has not been examined previously.

The results of the present study indicate that depression is the only aspect of distress associated with physical activity. Depression might be characterized by symptoms such as sadness, changes in sleep, lack of initiative and withdrawal from social contact. This might affect patients' quality of life, leisure- and social activities ${ }^{[29]}$. In the present study, patients who were depressed at baseline walked 2789 fewer steps (median) per day compared to patients without depression. This equal $28 \%$ of the daily 10,000 steps recommended for healthy adults ${ }^{[30]}$. Although steps are counted in various activities and settings, an objective registration of number of steps, like in the present study, contains no information about the type of physical activity nor the context associated with walking steps ${ }^{[31]}$. Previous research has indicated that leisure physical activity is associated with lower prevalence of depression and work- related physical activity with greater prevalence. Context might therefore be an important factor to consider when investigating the association between depression and physical activity ${ }^{[27]}$.

We might only speculate why the association between baseline walking steps and depression six weeks later was not maintained. Physical fit patients tend to be more physically active ${ }^{[32]}$, and whether patients is physically active at diagnosis is a strong predictor of whether the patient will continue being physically active during oncological treatment ${ }^{[8]}$. Still, many cancer patients reduce their physical activity levels after diagnosed with cancer ${ }^{[7]}$. Although we do not have information about physical activity at six weeks, we know that patients undergoing oncological treatment, like in the present study, might reduce their activity levels ${ }^{[33]}$. Treatment may also impact distress ${ }^{[3]}$, which might reflect the increase in levels of depression in the present study from baseline to six weeks. However, knowing that distress tends to be at its highest at the time of diagnosis ${ }^{[3]}$, it was somewhat surprising that only $7 \%$ of the patients were depressed at baseline. With no increase at six weeks the prevalence was lower than the $15 \%$ prevalence reported by others ${ }^{[34]}$.

For depression and moderate- to- vigorous intensity physical activity we found a tendency pointing in the same direction as number of steps at baseline. This might indicate that the intensity of physical activity also seems to be 
of importance. Using objective recordings is considered to provide valid and reliable measures of physical activity levels ${ }^{[35]}$. Still, wearing an activity monitor might motivate some patients to increase their activity level resulting in changed habitual physical activity behavior during the period when data was collected. On the other side, some patients might not wear the activity monitor, resulting in underestimations of actual physical activity ${ }^{[36]}$.

Our results might imply that being physically active, such as when walking, seems to be of importance for depression for cancer patients at the beginning of oncological treatment. Walking might be a convenient mode of physical activity at the start of treatment as it can be implemented in everyday activities at a time where physicaland psychosocial barriers might contribute to reduce activity levels ${ }^{[37]}$. Walking interventions have been conducted among cancer patients during treatment and have proven to be feasible and effective in reducing emotional distress ${ }^{[38]}$.

Our findings might suggest that it could be beneficial to present cancer patients with information about physical activity before the start of oncological treatment. The time between diagnosis and start of treatment is a time when patients are particularly open to information concerning a healthy lifestyle, especially if the information is presented by health care professionals ${ }^{[39]}$. Information about physical activity might be especially important since some patients might restrict their physical activity due to their treatment ${ }^{[33]}$ and uncertainty of how active they can be ${ }^{[40]}$. We recommend in future studies to examine how the method used to assess physical activity might affect the association between physical activity and distress, especially psychological distress.

The main strength of the present study was the use of objectively recorded moderate- to- vigorous intensity physical activity and walking steps, as this strengthens the internal validity of the study and are more valid and reliable than self- reports. Strength is also a heterogeneous sample of patients with regard to cancer diagnosis and treatment type. Limitations are lack of sample size calculation with respect to the present study, few patients with symptoms of depression, use of convenience sampling and lack of individual information about the type of treatment and severity of disease as shown relevant for levels of distress and physical activity ${ }^{[3,33]}$. The activity monitor has limitations for use during activities in water as it is not waterproof. Only one day of objectively recorded physical activity data does not necessarily reflect a person's habitual physical activity level. No conclusion can be drawn regarding the direction of causality in our results, due to our study design and we cannot rule out that factors other than physical activity may influence depression in the beginning of oncological treatment.

In conclusion, an inverse association between physical activity and depression at baseline was found, especially in relation to number of walking steps. This finding must be interpreted with caution due to few patients with depression. Whether a physically active lifestyle prevents or reduces distress when diagnosed with cancer has to be elaborated in future studies.

\section{CONCLUSION}

In conclusion, an inverse association between physical activity and depression at baseline was found, especially in relation to number of walking steps. This finding must be interpreted with caution due to few patients with depression. Whether a physically active lifestyle prevents or reduces distress when diagnosed with cancer has to be elaborated in future studies.

\section{DECLARATION OF CONFLICTING INTEREST}

The authors have no conflicts of interest (political, personal, religious, ideological, academic, intellectual, commercial or any other) to declare in relation to this manuscript. 


\section{FUNDING}

This study is funded by the Grieg Foundation and the Norwegian Cancer Society.

\section{REFERENCES}

1. Pinto, B.M. and J.J. Trunzo, Health behaviors during and after a cancer diagnosis. Cancer, 2005. 104(11 Suppl): p. 2614-23.

2. Zabora, J., et al., The prevalence of psychological distress by cancer site. Psychooncology, 2001. 10(1): p. 19-28.

3. Carlson, L.E., et al., What goes up does not always come down: patterns of distress, physical and psychosocial morbidity in people with cancer over a one year period. Psychooncology, 2013. 22(1): p. 168-76.

4. Thorsteinsdottir, T., et al., Intrusive thoughts and quality of life among men with prostate cancer before and three months after surgery. Health Qual Life Outcomes, 2013. 11: p. 154.

5. Brunet, J., et al., Stress and physical activity in young adults treated for cancer: the moderating role of social support. Support Care Cancer, 2014. 22(3): p. 689-95.

6. Schmitz, K.H., et al., American College of Sports Medicine roundtable on exercise quidelines for cancer survivors. Med Sci Sports Exerc, 2010. 42(7): p. 1409-26.

7. Courneya, K.S., K.H. Karvinen, and J.K.H. Vallance, Exercise Motivation and Behavior Change, in Handbook of Cancer Survivorship, M. Feuerstein, Editor. 2007, Springer US. p. 113-132.

8. Courneya, K.S. and C.M. Friedenreich, Relationship between exercise pattern across the cancer experience and current quality of life in colorectal cancer survivors. J Altern Complement Med, 1997. 3(3): p. 215-26.

9. Salmon, P., Effects of physical exercise on anxiety, depression, and sensitivity to stress: a unifying theory. Clin Psychol Rev, 2001. 21(1): p. 33-61.

10. Sabiston, C.M. and J. Brunet, Reviewing the Benefits of Physical Activity During Cancer Survivorship. American Journal of Lifestyle Medicine, 2012. 6(2): p. 167-177.

11. Knobf, M.T., R. Musanti, and J. Dorward, Exercise and quality of life outcomes in patients with cancer. Semin Oncol Nurs, 2007. 23(4): p. 285-96.

12. Stagl, J.M., et al., Postsurgical physical activity and fatigue-related daily interference in women with non-metastatic breast cancer. Psychol Health, 2014. 29(2): p. 177-98.

13. Jacobsen, P.B., et al., Effects of self-directed stress management training and home-based exercise on quality of life in cancer patients receiving chemotherapy: a randomized controlled trial. PsychoOncology, 2013. 22(6): p. 1229-1235.

14. Phillips, S.M. and E. McAuley, Associations between self-reported post-diagnosis physical activity changes, body weight changes, and psychosocial well-being in breast cancer survivors. Support Care Cancer, 2014.

15. Jones, S.M., et al., Depression and quality of life before and after breast cancer diagnosis in older women from the Women's Health Initiative. J Cancer Surviv, 2015.

16. Branstrom, R., et al., Physical activity following a breast cancer diagnosis: Implications for self-rated health and cancer-related symptoms. Eur J Oncol Nurs, 2015.

17. Arving, C., et al., Early rehabilitation of cancer patients - a randomized controlled intervention study. BMC Cancer, 2013. 13: p. 9. 
18. Zigmond, A.S. and R.P. Snaith, The hospital anxiety and depression scale. Acta Psychiatr Scand, 1983. 67(6): p. 361-70.

19. Saboonchi, F., et al., Examination of the construct validity of the Swedish version of Hospital Anxiety and Depression Scale in breast cancer patients. Quality of Life Research, 2013. 22(10): p. 2849-2856.

20. Horowitz, M., N. Wilner, and W. Alvarez, Impact of Event Scale: a measure of subjective stress. Psychosom Med, 1979. 41(3): p. 209-18.

21. Horowitz, M.J., N.P. Field, and C.C. Classen, Stress response syndromes and their treatment, in Handbook of stress: Theoretical and clinical aspects (2nd ed.), L.G.S. Breznitz, Editor. 1993, Free Press: New York, NY, US. p. 757-773.

22. Joseph, S., Psychometric evaluation of Horowitz's Impact of Event Scale: a review. J Trauma Stress, 2000. 13(1): p. 101-13.

23. Berntsen, S., et al., Validity of physical activity monitors in adults participating in free-living activities. Br J Sports Med, 2010. 44(9): p. 657-64.

24. Cereda, E., et al., Assessing energy expenditure in cancer patients: a pilot validation of a new wearable device. JPEN J Parenter Enteral Nutr, 2007. 31(6): p. 502-7.

25. Garber, C.E., et al., American College of Sports Medicine position stand. Quantity and quality of exercise for developing and maintaining cardiorespiratory, musculoskeletal, and neuromotor fitness in apparently healthy adults: guidance for prescribing exercise. Med Sci Sports Exerc, 2011. 43(7): p. 1334-59.

26. Hosmer, D.W. and S. Lemeshow, Applied logistic regression (2nd Edition). 2000, New York, NY, USA: John Wiley \& Sons.

27. McKercher, C.M., et al., Physical activity and depression in young adults. Am J Prev Med, 2009. 36(2): p. 161-4.

28. Vallance, J.K., et al., Accelerometer-assessed physical activity and sedentary time among colon cancer survivors: associations with psychological health outcomes. J Cancer Surviv, 2015. 9(3): p. 404-11.

29. Craft, L.L., et al., Exercise effects on depressive symptoms in cancer survivors: a systematic review and meta-analysis. Cancer Epidemiol Biomarkers Prev, 2012. 21(1): p. 3-19.

30. Tudor-Locke, C. and D.R. Bassett, Jr., How many steps/day are enough? Preliminary pedometer indices for public health. Sports Med, 2004. 34(1): p. 1-8.

31. Choi, B.C., et al., Daily step goal of 10,000 steps: a literature review. Clin Invest Med, 2007. 30(3): $p$. E146-51.

32. Shang, J., et al., Who will drop out and who will drop in: exercise adherence in a randomized clinical trial among patients receiving active cancer treatment. Cancer Nurs, 2012. 35(4): p. 312-22.

33. Kwan, M., et al., Change in physical activity during active treatment in a prospective study of breast cancer survivors. Breast Cancer Research and Treatment, 2012. 131(2): p. 679-690.

34. Nordin, K., et al., Predicting anxiety and depression among cancer patients: a clinical model. Eur J Cancer, 2001. 37(3): p. 376-84.

35. Dishman, R.K., R.A. Washburn, and D.A. Schoeller, Measurement of Physical Activity. Quest, 2001. 53(3): p. 295-309.

36. Pedisic, Z. and A. Bauman, Accelerometer-based measures in physical activity surveillance: current practices and issues. Br J Sports Med, 2015. 49(4): p. 219-223.

37. Morris, J.N. and A.E. Hardman, Walking to health. Sports Med, 1997. 23(5): p. 306-32. 
38. Mock, V., et al., Fatigue and quality of life outcomes of exercise during cancer treatment. Cancer Practice, 2001. 9(3): p. 119-127.

39. Stull, V.B., D.C. Snyder, and W. Demark-Wahnefried, Lifestyle interventions in cancer survivors: designing programs that meet the needs of this vulnerable and growing population. J Nutr, 2007. 137( 1 Suppl): p. 243S-248S.

40. Tonosaki, A. and M. Ishikawa, Physical activity intensity and health status perception of breast cancer patients undergoing adjuvant chemotherapy. Eur J Oncol Nurs, 2014. 18(2): p. 132-9. 


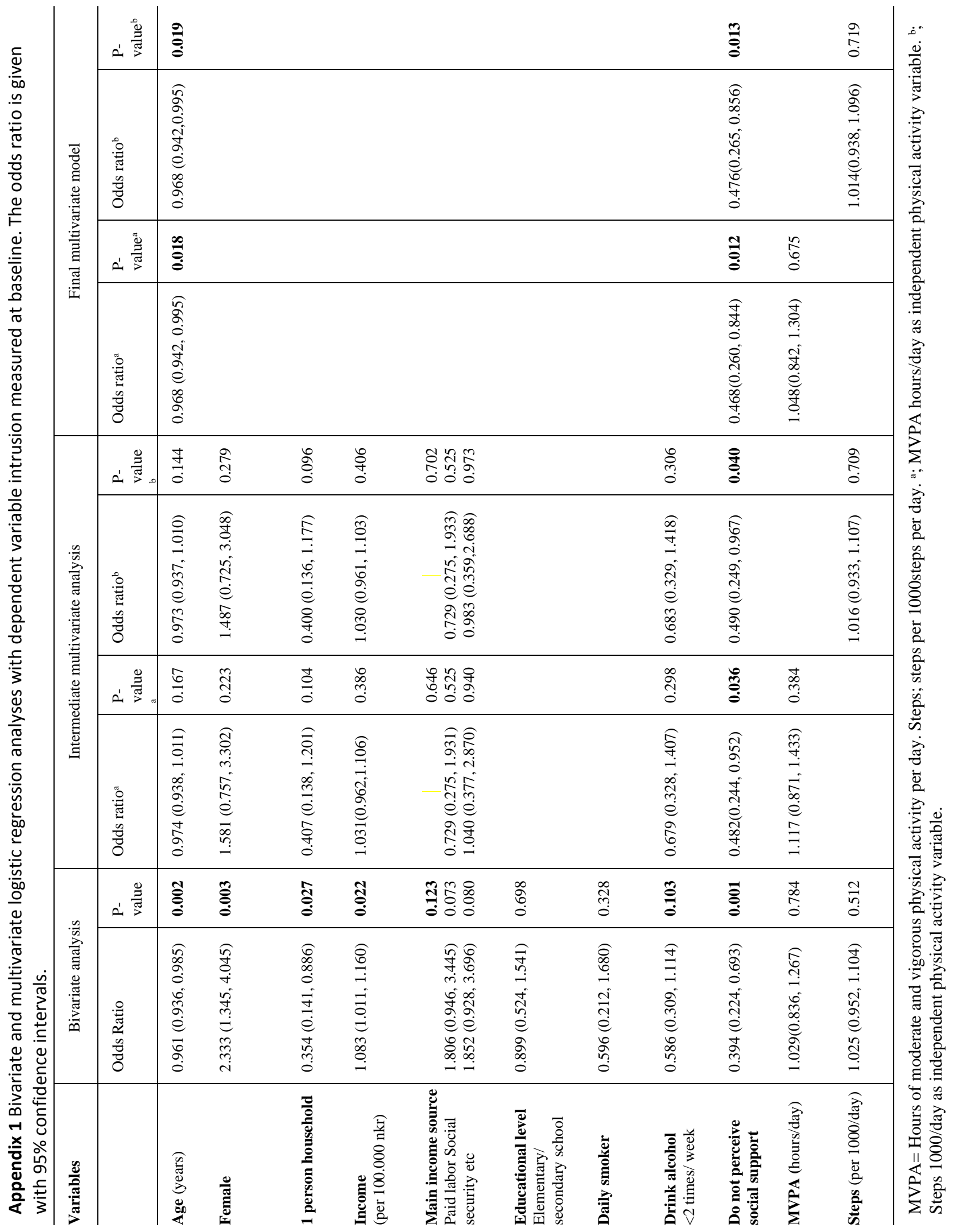




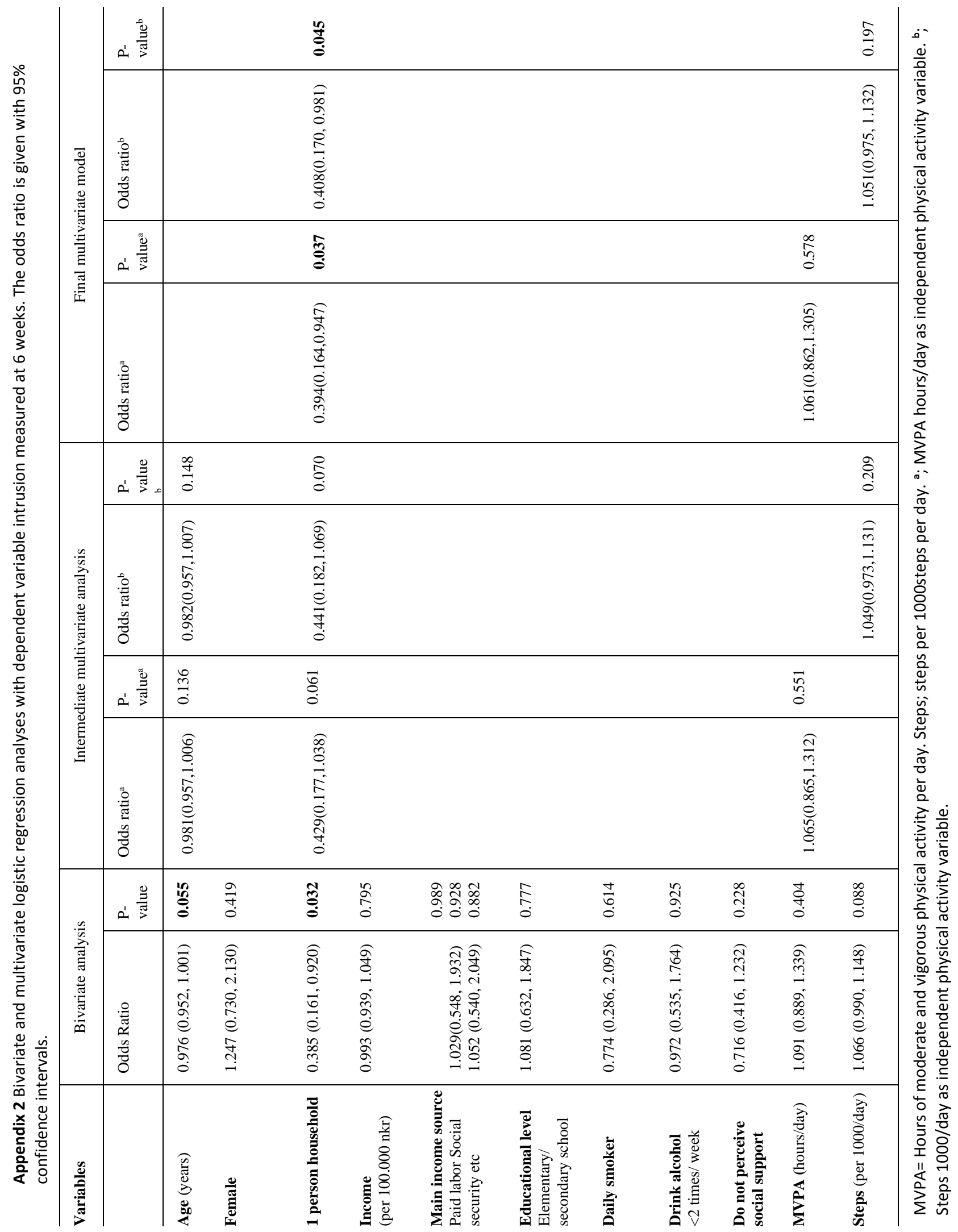




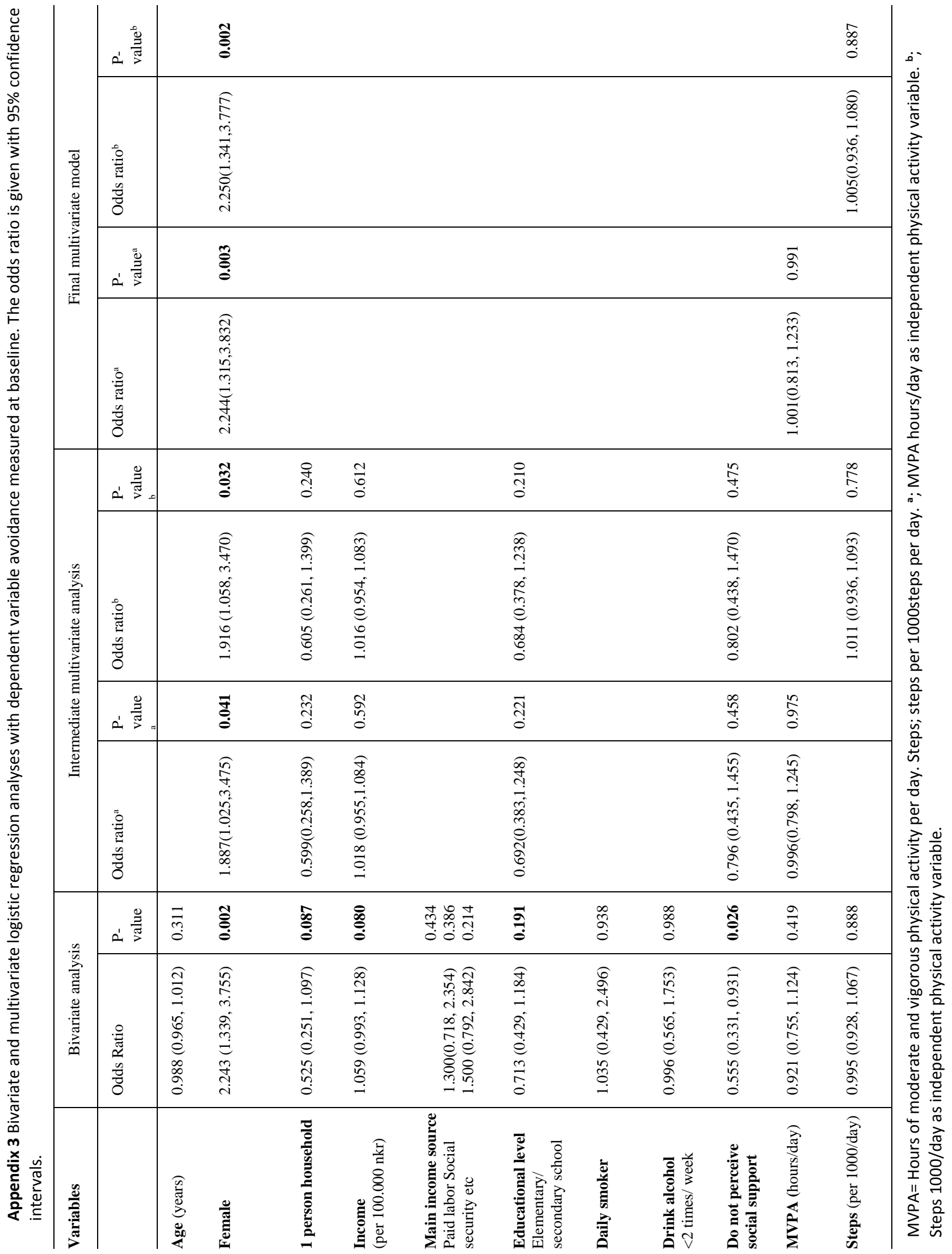




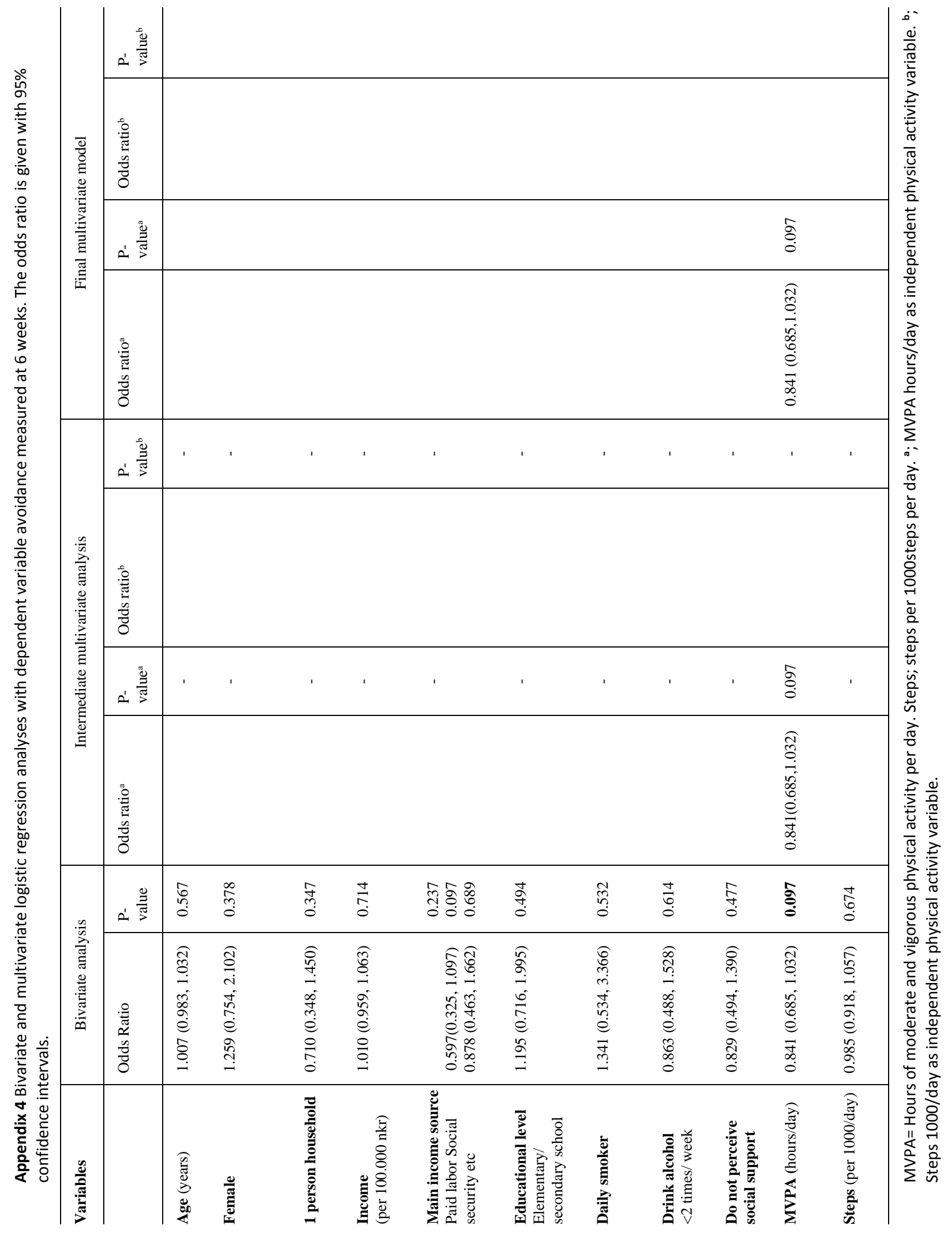




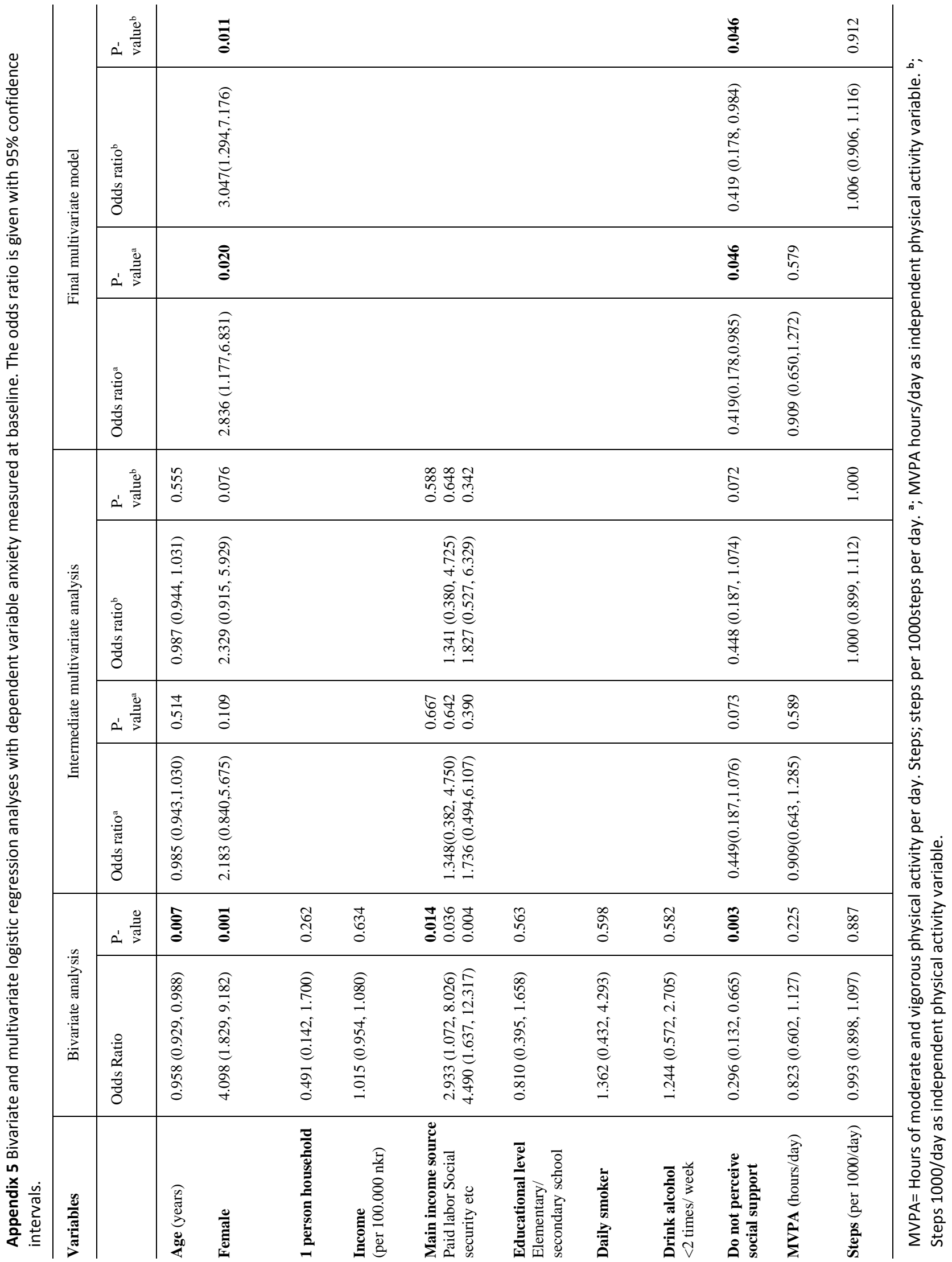




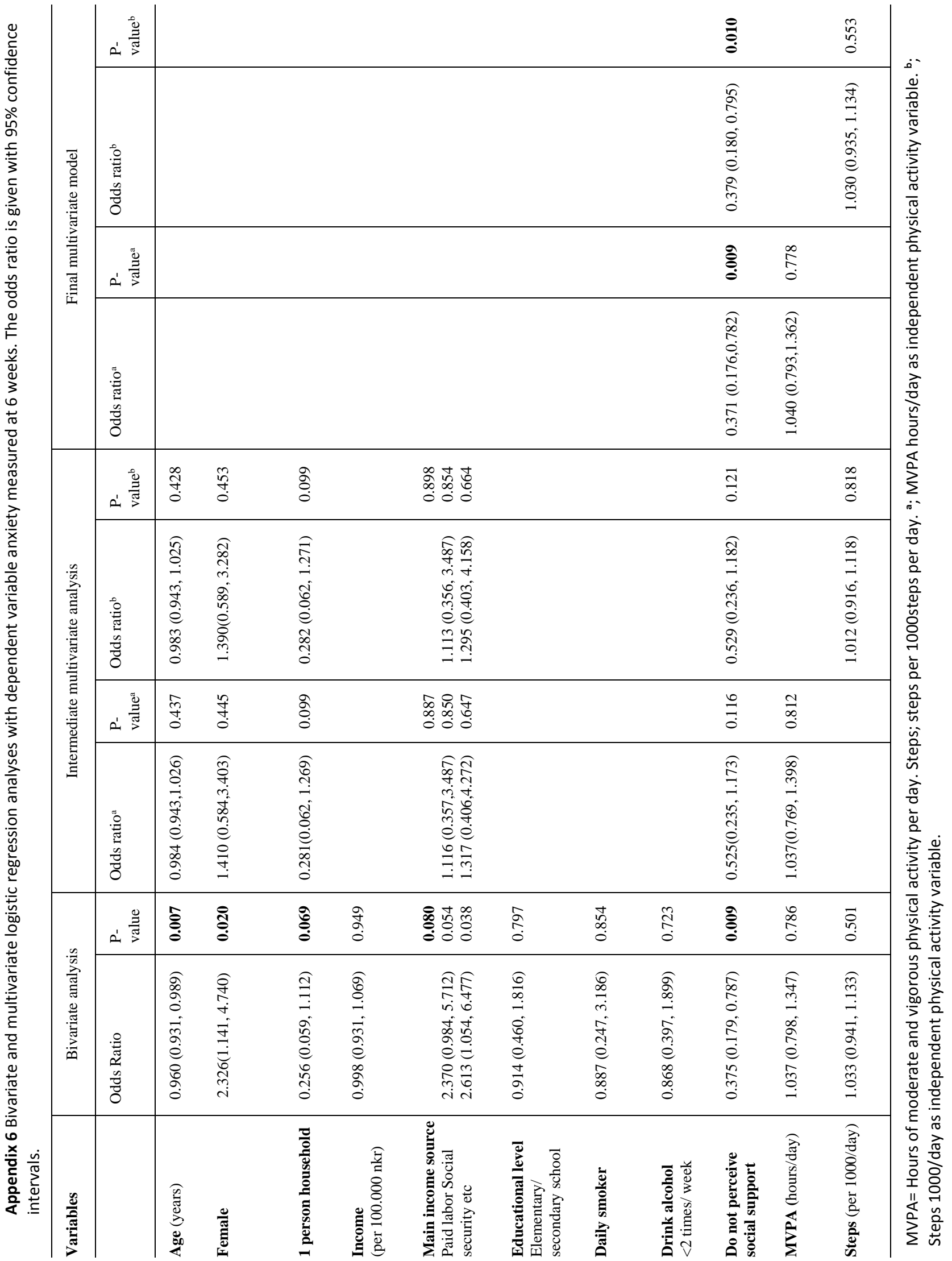

